\title{
HYPOTHALAMIC HAMARTOMA AND GENERALIZED EPILEPSY
}

The evolution of symptomatic generalized epilepsy was studied in 12 of 20 children with hypothalamic hamartoma who were treated surgically by transcallosal microsurgical resection at the Royal Children's Hospital, Parkville, Australia. Intraoperative EEGs were recorded from the hamartoma and simultaneously from the scalp and frontal cortex before, during, and after resection in 7 patients. Gelastic seizures in the 12 patients studied began on average at 6 months of age, complex partial and focal motor seizures at 4 years, and tonic seizures at 6 years. Scalp EEGs were normal in one third of patients at first, even after epilepsy was diagnosed. Epileptiform EEG generalized slow spike-wave patterns developed later with tonic seizures. Interictal spike-wave was recorded intraoperatively over scalp and cortex but not from the hamartoma. All 12 had developmental disability and behavioral disorders. The triad of refractory generalized seizures, slow SW in EEG, and neurobehavioral disorder was reversible after resection of the hamartoma. Tonic generalized seizures ceased in 11 of 12 patients, interictal SW activity was markedly reduced, and behavior improved, but the remission of seizures and decrease in SW activity were delayed up to 6 months in some cases. Gelastic seizures arise from the intrinsically epileptogenic hamartoma itself, whereas the interictal spike-wave and generalized seizures have an extralesional origin. The evolution of EEG abnormalities and generalized seizures may reflect a secondary epileptogenesis. (Freeman JL, Harvey AS, Rosenfeld JV et al. Generalized epilepsy in hypothalamic hamartoma. Evolution and postoperative resolution. Neurology March (1 of 2) 203;60:762-767). (Reprints: Dr Jeremy L Freeman, Department of Neurology, Royal Children's Hospital, Flemington Road, Parkville VIC 3052, Australia).

COMMENT. Symptomatic generalized epilepsy and neurobehavioral disorders often complicate and follow the onset of gelastic seizures in children with hypothalamic hamartoma. The generalized seizures, associated slow spike-wave EEG, and behavior problems are usually reversible following resection of the hamartoma. Since antiepileptic drugs are largely ineffective, early diagnosis and operation before the development of generalized seizures and behavioral deterioration should be considered. It is noteworthy that one report, cited by the authors, has attributed the symptomatic generalized seizures to either widespread occult cerebral dysgenesis or irreversible cerebral damage (Sisodiya SM et al. Epilepsia 1997;38:1008-1010). The present study emphasizes the functional nature and reversibility of generalized seizures complicating hypothalamic hamartoma.

\section{POKEMON PHENOMENON AND SEIZURES}

A survey by questionnaire of 67 Japanese children affected by watching the animated television series Pocket Monsters (Pokemon) on December 16, 1997 and their parents was compared with that of children not affected by the program, in a study conducted at Showa University School of Medicine, Tokyo, Japan, and other centers. Children who visited 14 pediatric clinics for other reasons within 2 months after the incident were included. Of 1,373 replies, 800 were male and 558 female ( 15 unspecified), and mean age was $6.8+/-3.5$ years. The majority $(80 \%)$ had watched the program, and 67 $(6.1 \%$; 40 males, 27 females) were affected. Ten had photosensitive seizures $(0.9 \% ; 4$ 